\title{
B meson decays from moving-NRQCD on fine MILC lattices
}

\section{T. H. Davies and K. Y. Wong*}

Department of Physics and Astronomy, University of Glasgow, Glasgow, G12 8QQ, United Kingdom

\section{G. P. Lepage}

Laboratory of Elementary Particle Physics, Cornell University, Ithaca, New York 14853, USA

Lattice simulations of $B \rightarrow \pi l v$ decays are problematic in the low $q^{2}$ region when the pion has to have large momentum in the lattice frame for a $B$ meson at rest or moving slowly. The movingNRQCD formalism provides a way around this by giving an accurate discretization of a $b$ quark moving at arbitrary velocity $v$, and therefore a $B$ meson moving at high momentum in the lattice frame. Here we show results from simulations of the moving-NRQCD action complete through $O(1 / M)$ coupled to the asqtad action on a MILC fine ensemble. We show $B, B^{*}$ masses as a function of $v$ and demonstrate how to determine the meson kinetic mass and the renormalization of $v$ non-perturbatively. We will also discuss the perturbative renormalization of the movingNRQCD action from high- $\beta$ Monte Carlo simulations.

XXIVth International Symposium on Lattice Field Theory

July 23-28, 2006

Tucson, Arizona, USA

\footnotetext{
*Speaker.
} 


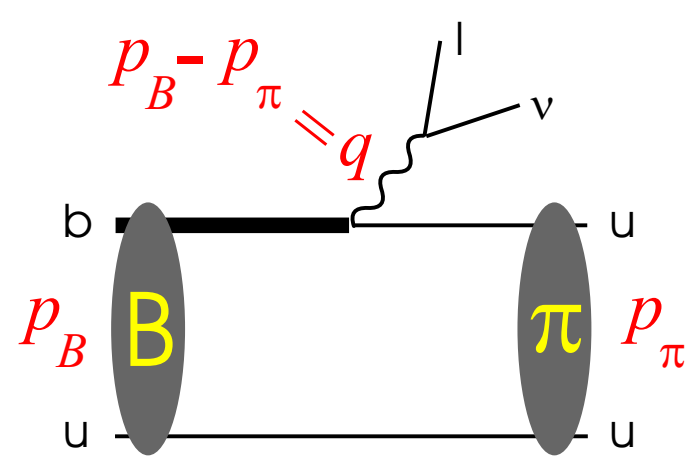

Figure 1: Kinematics of $B \rightarrow \pi l v$ semileptonic decay. $p_{B}$ is the momentum of the $B$ meson and $p_{\pi}$ is the momentum of the pion, $q$ is the momentum transfer.

\section{INTRODUCTION}

Precise determination of the Cabibbo-Kobayashi-Maskawa (CKM) matrix is crucial for high precision tests of the standard model. The matrix element $\left|V_{u b}\right|$ can be determined from studies of $B \rightarrow \pi l v$ semileptonic decays in B-factories. Such decays, however, involve strong interaction dynamics also in the form of hadronic matrix elements; therefore precise determination of $\left|V_{u b}\right|$ requires accurate calculation of these form factors.

Lattice QCD provides a first principles non-perturbative approach for calculating the form factors $f_{+}\left(q^{2}\right), f_{0}\left(q^{2}\right)$ in $B \rightarrow \pi l v$ decays. Fig. 11 shows the kinematics of the process. Lattice simulations are problematic, however, when the pion has very large recoil momentum, i.e., when $q^{2}$ is small. This is because discretization errors increase as the pion momentum increases; and statistical errors become worse when the hadrons have large momenta. Lattice results are therefore available at the large $q^{2}$ region only [1]. These simulations were done in the $B$ meson rest frame, and two different techniques were used to handle the $b$ quark on the lattice: non-relativistic QCD (NRQCD) and the Fermilab formalism. Because of the lack of data at small $q^{2}$ error in $\left|V_{u b}\right|$ is dominated by error in lattice results.

This problem can be solved in two stages. First instead of working in the $B$ meson rest frame we choose a moving frame in which the $B$ meson is moving in the opposite direction of the pion. This reduces the momentum of the pion, but at the expense of increasing finite-lattice-spacing errors for the now high-momentum $B$ meson. The increased errors in the $B$ system are mostly associated with the $b$ quark since most of its momentum is carried by the heavy quark. We work around this problem by using the moving-NRQCD (mNRQCD) formalism [2, 3] for the $b$ quark.

Moving-NRQCD provides an accurate discretization of a heavy quark moving at arbitrary velocity $v$. It is useful to parameterize the $b$ quark momentum as $p_{b}=u m_{b}+k$ where $u=p_{B} / m_{B}=$ $\left(E_{B} / m_{B}, v\right)$ is the 4-velocity of the $B$ meson. The residual momentum $k$ is much smaller than $p_{B}$ and $k^{2} \sim O\left(\Lambda_{Q C D}^{2}\right)$. In mNRQCD one treats the velocity $v$ exactly and discretizes the residual momentum only. The leading discretization errors therefore become $O\left(a^{2} k^{2}\right)$ instead of $O\left(a^{2} p_{B}^{2}\right)$, which could be as large as several $\mathrm{GeV}^{2}$. We refer the readers to Refs. [2, 3] for details of the formulation.

The mNRQCD action can be derived using the FWT transformation or by boosting the NRQCD 
action to a moving frame [2, 3]. In this project we use an action which is complete through $O(1 / M)$ and tadpole improved (the tadpole factor $u_{0}$ is implicit in the equations):

Evolution equation:

$$
G_{x, t+1}=\left(1-\frac{\delta H}{2}\right)\left(1-\frac{\delta H_{0}}{2 n}\right)^{n} U_{x, \hat{t}}^{\dagger}\left(1-\frac{\delta H_{0}}{2 n}\right)^{n}\left(1-\frac{\delta H}{2}\right) G_{x, t}
$$

Action :

$$
\begin{aligned}
H_{0}= & -i v \cdot \Delta^{ \pm}-\frac{\Delta^{(2)}}{2 M_{0} \gamma} \\
\delta H= & -\frac{1}{2 M_{0} \gamma} \sigma \cdot \tilde{B}_{m}+\left(\frac{1}{2 M_{0} \gamma}+\frac{1}{4 n}\right)\left(v \cdot \Delta^{ \pm}\right)^{2}-\frac{i}{4 M_{0} n \gamma}\left(v \cdot \Delta^{ \pm}\right) \Delta^{(2)} \\
& +\frac{i}{6} \sum_{i} v_{i} \Delta_{i}^{+} \Delta_{i}^{ \pm} \Delta_{i}^{-}+\frac{1}{24 M_{0} \gamma} \sum_{i} \Delta_{i}^{(4)}-\frac{1}{6 M_{0} \gamma}\left(v \cdot \Delta^{ \pm}\right) \sum_{i} v_{i} \Delta_{i}^{+} \Delta_{i}^{ \pm} \Delta_{i}^{-} \\
\tilde{B}_{m}= & \gamma\left(\tilde{B}-v \times \tilde{E}-\frac{\gamma}{\gamma+1} v(v \cdot \tilde{B})\right)
\end{aligned}
$$

where $\gamma=1 / \sqrt{1-v^{2}}, M_{0}$ is the input bare mass, $n$ is the stability parameter, and $\tilde{B}_{m}$ is the magnetic field in the moving frame (we use $O\left(a^{2}\right)$ improved electric field $\tilde{E}$ and magnetic field $\tilde{B}$ ). The corresponding dispersion relation is given by

$$
E(k)=E_{0}+\sqrt{P_{\text {tot }}^{2}+M_{k i n}^{2}}=E_{0}+\sqrt{\left(Z_{p} P_{0}+k\right)^{2}+Z_{m}^{2} M_{0}^{2}}
$$

where $P_{0}=\gamma M_{0} v$. Here $E_{0}$ is the zero energy, $Z_{m}$ is the mass renormalization and $Z_{p}$ is the external momentum renormalization, which is a new feature in $\mathrm{MNRQCD}$. These renormalization constants have perturbative expansions

$$
\begin{aligned}
E_{0}(v) & =c_{1, E 0}(v) \alpha_{s}+\ldots, \\
Z_{m}(v) & =1+c_{1, Z m}(v) \alpha_{s}+\ldots, \\
Z_{p}(v) & =1+c_{1, Z p}(v) \alpha_{s}+\ldots
\end{aligned}
$$

Notice the dependence on the velocity. In the next section we will discuss how to extract the perturbative coefficients $c_{1}, c_{2} \ldots$ from high- $\beta$ Monte Carlo simulations.

\section{PERTURBATIVE EXPANSIONS FROM HIGH- $\beta$ (WEAK COUPLING) SIMULATIONS}

Knowing how the bare action parameters renormalize on the lattice is crucial for accurate simulations. The first order coefficients (quenched with the Wilson plaquette action, $\delta H=0$ ) have been computed in standard lattice perturbation theory using diagrammatic techniques [ 4 ]. Analytic calculations, however, are very challenging because of the proliferation of diagrams due to the lattice cutoff. This is particularly true at higher orders. An alternative is to use numerical methods. Here we determine the first order coefficients from high- $\beta$ simulations [\$] ] and compare the results to the analytic calculations. 

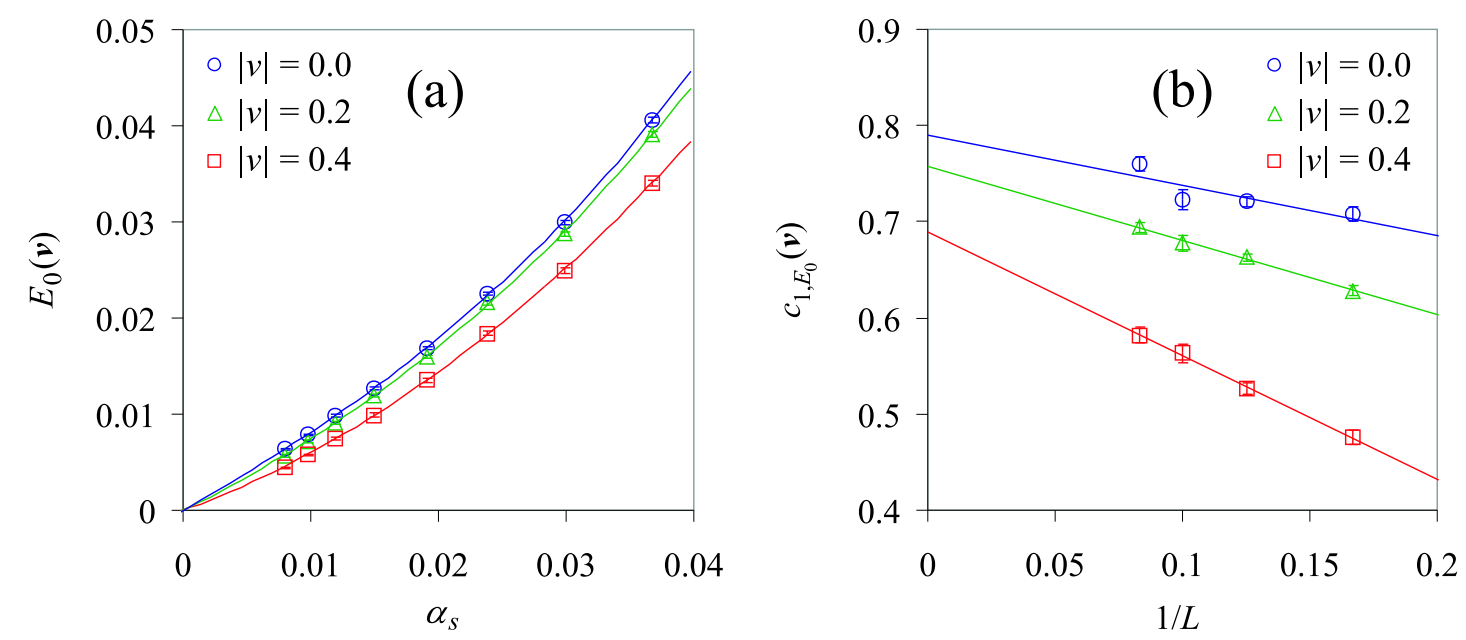

Figure 2: (a) Zero energy $E_{0}(v)$ as a function of $\alpha_{s}$. The volume is $6^{3} \times 12$. (b) $1 / L$ infinite volume extrapolation of the first order coefficients $c_{1, E 0}(v)$.

We start by doing ten sets of simulations, at volumes $V=L^{3} \times T=6^{3} \times 12,\left(8^{3}, 10^{3}, 12^{3}\right) \times 16$, with $\beta$-values range from 10.0 to 60.0. The corresponding $\alpha_{s}$ are of the order of 0.01-0.1. It is important to work at small couplings so that the theory enters the perturbative phase. Twisted boundary conditions are also used in the $x$ and $y$ directions to suppress non-perturbative effects due to $Z(3)$ phases [5]. In each simulation we measure the heavy quark energy at several momenta and fit the results to Eq. 1.2 to extract the renormalization constants. We were able to work at small momentum, $k^{2}<0.3$, by using generalized boundary conditions for the quark field in the $z$ direction [6]. Finally to obtain the perturbative coefficients we fit the renormalization constants obtained at different $\beta$ to a polynomial in $\alpha_{s}$.

Fig. Za plots the zero energy $E_{0}(v)$ against $\alpha_{s}$ at several velocities at volume $6^{3} \times 12$. The "slopes" of the lines are the first order coefficients $c_{1, E 0}(v)$ and the "curvatures" are the second order coefficients. Fig. $2 \mathrm{~b}$ shows the $1 / L$ infinite volume extrapolation of the first order coefficients $c_{1, E 0}(v)$; and the final results are given in Fig. 3. Our numbers agree very well with the analytic results (given by the curve in the graph). We have also checked that reversing the fit order, i.e., extrapolate to infinite volume at each $\beta$ and then fit to an expansion in $\alpha_{s}$, gives consistent results.

Fig. $⿴$ shows the external momentum renormalization $Z_{p}(v)$ obtained at $6^{3} \times 12$ and $12^{3} \times 16$. Despite the large statistical errors all the data are consistent with 1 . We can conclude that there is little or no renormalization of the bare external momentum. This is consistent with the fact that $v$ is protected from renormalization because of approximate re-parameterization invariance of the action [2, 3].

\section{3. $B$ MESON DECAYS ON MILC FINE LATTICES}

In this section we present results for $B$ meson correlators on MILC fine lattices. We study the dependence of $B$ and $B^{*}$ masses on $v$. We also show how to extract the momentum renormalization from simulations. 


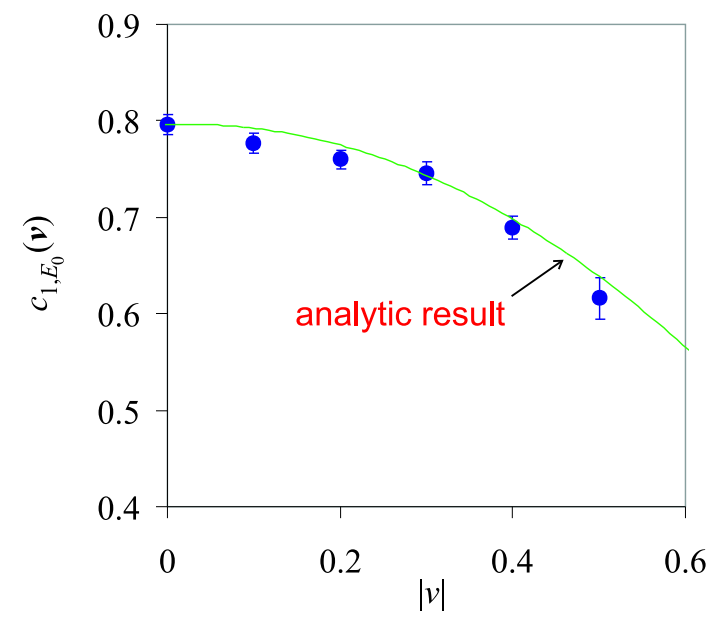

Figure 3: Zero energy first order perturbative coefficients $c_{1, E 0}(v)$ after infinite volume extrapolation. The curve shows the analytic results obtained in Ref. 沟.
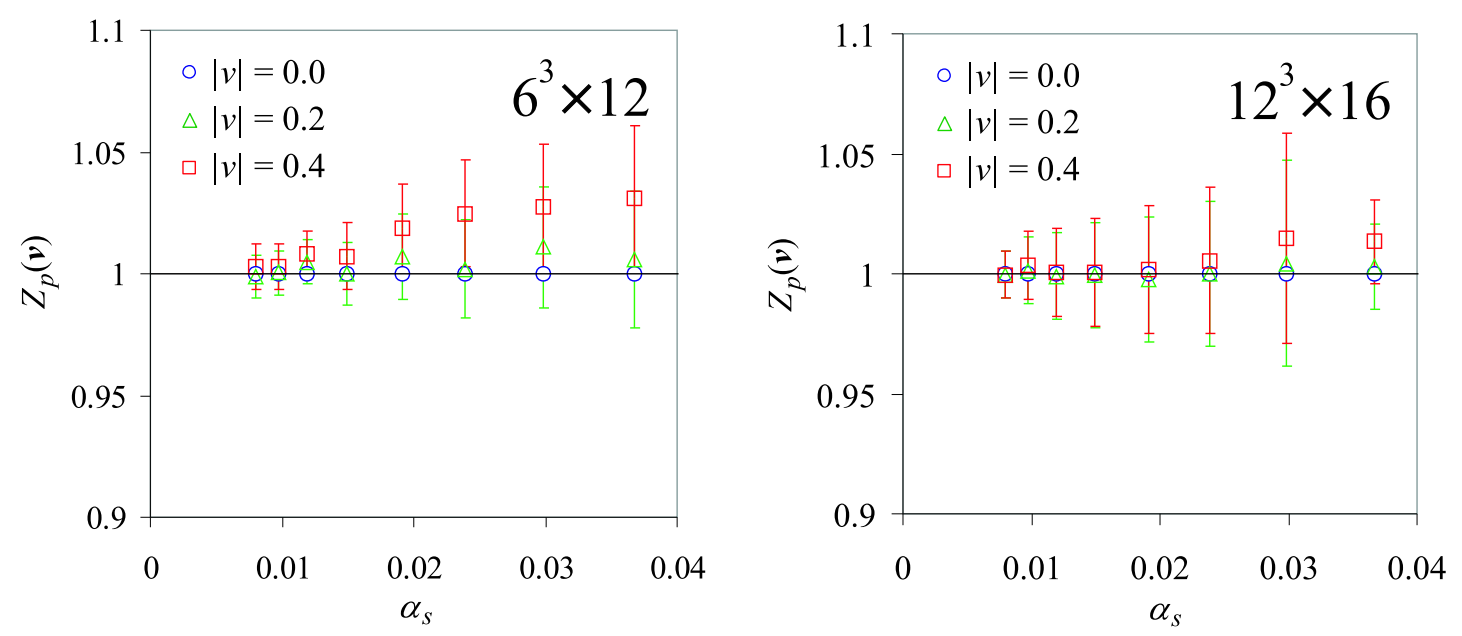

Figure 4: External momentum renormalization $Z_{p}(v)$ as a function of $\alpha_{s}$. Results are shown for volumes $6^{3} \times 12$ and $12^{3} \times 16$.

Simulations were done with the $O(1 / M)$ accurate tadpole improved $\left(u_{0}=0.8541\right)$ mNRQCD action at three different velocities $v=\left(v_{x}, 0,0\right), v_{x}=0.0,0.25$ and 0.5. The MILC fine lattices have a lattice spacing of $2.258 \mathrm{GeV}^{-1}$, which is set by the $\Upsilon 2 \mathrm{~S}-1 \mathrm{~S}$ mass splitting [ [7]; the sea quark mass is $u_{0} a m_{f_{1,2}}=0.0062,0.031$. The bare mass is set to $a M_{0}=1.95(n=2)$ for the heavy quark; and we use the asqtad formalism for valence light quarks with a quark mass $a m_{q}=0.031$. It has been shown that, from previous NRQCD studies on heavy-light systems and the $\Upsilon$ spectrum, these quark masses reproduce the correct $B_{s}$ meson mass at $v=0$. Gaussian smearings with a radius of $2 a$ were applied to the heavy quark at both the source and the sink. Once again we measure the meson energy at different momenta, and fit the results to Eq. 1.2 to extract the zero energy $E_{0}(v)$, kinetic mass $m_{\text {kin }}(v)$, and momentum renormalization $Z_{p}(v)$. Thirty nine different momenta, with 

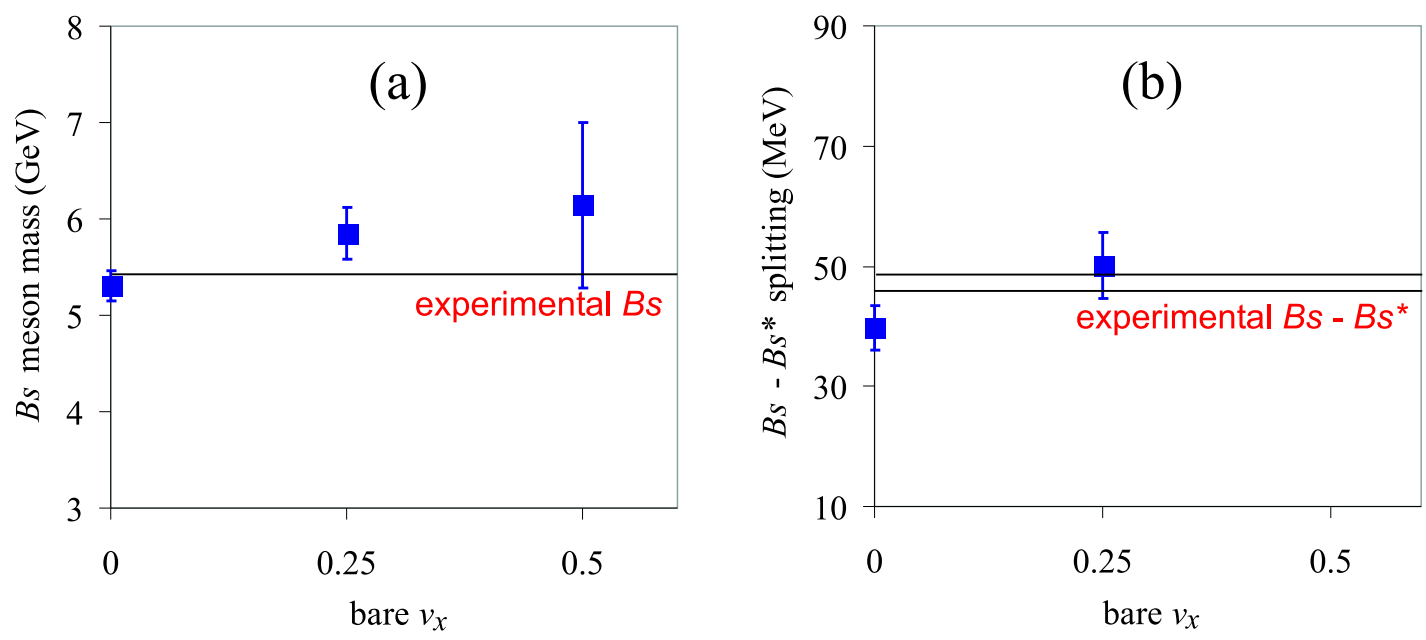

Figure 5: (a) $B_{s}$ meson mass $m_{k i n}$ as a function of bare velocity. (b) $B_{s}-B_{s}^{*}$ energy splitting as a function of $v$. The experimental values are given by the horizontal lines.

$0 \leq k^{2} \leq 0.45$, were used.

Fig. 5a plots the $B_{s}$ mass as a function of bare velocity $v_{x}$. Result at $v=0$ agrees well with the experimental value as suggested in the last paragraph. Our data show almost no dependence on the velocity, although a small increase can be observed as $v_{x}$ becomes large. This suggests that one may need to tune (reduce) the input bare mass $M_{0}$ as $v$ increases.

The energy splitting of $B_{s}-B_{s}^{*}$ is plotted in Fig. $5 \mathrm{~b}$. Again results show only a small velocity dependence. Data at $v=0$, however, is about one $\sigma$ lower than the experimental value. It is believed that radiative correction of the $\sigma \cdot B$ term in the action is responsible for this deviation from the experimental data.

Finally the external momentum renormalization $Z_{p}(v)$ for $B_{s}$ meson is given in Fig. 6. All the data points are consistent with 1 . This agrees with the results obtained in perturbation theory (see Fig. 7 in the last section), and again reflects the re-parameterization invariance property of the action.

Fig. 5 and 6 both reveal that statistical errors increase significantly between $v_{x}=0.25$ and 0.5 . It is therefore advisable to use a more advanced smearing technique, such as the so-called "magic" smearing [3], in future simulations.

\section{CONCLUSION}

Moving-NRQCD provides a promising approach for studying semileptonic decays at high recoil on the lattice. This project represents a major step forward towards this goal. We have demonstrated that perturbative expansions can be computed in high- $\beta$ simulations with much less effort. First order results agree very well with the results obtained using analytic methods. Work is currently underway to extract higher-order coefficients from the data. In the second part of the paper we present results for $B$ meson correlators on MILC fine lattices. A variety of quantities have been measured at different $B$ meson velocities; and we observe little or no velocity dependence. This 


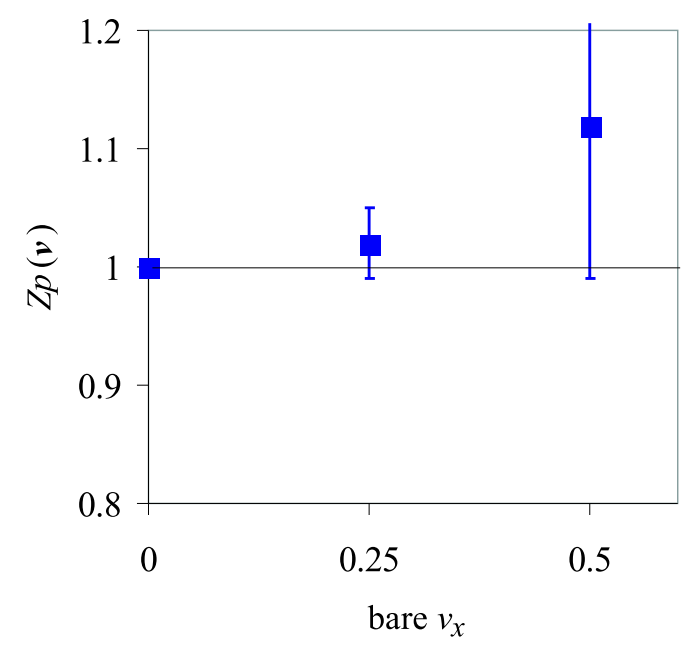

Figure 6: Momentum renormalization $Z_{p}(v)$ of $B_{s}$ as a function of input velocity.

is significant since it indicates that we do not need to tune the input bare parameters as $v$ changes. On the other hand our results show a sharp increase in statistical errors as $v$ becomes large. This suggests that it may be difficult to reach a very low $q^{2}$ value. It is, however, not necessary to give the form factors at very small $q^{2}$ since the main experimental focus is on $8 \mathrm{GeV}^{2} \leq q^{2} \leq 16 \mathrm{GeV}^{2}$. Simulations for $B \rightarrow \pi l v$ decays will start soon and we expect to have new results in the next year.

\section{ACKNOWLEDGMENTS}

This work was supported by PPARC (UK) and the DOE and NFS (USA). We thank the MILC Collaboration for making their unquenched gauge configurations available and the Fermilab Collaboration for use of their light propagators on the fine lattices. We thank Alex Dougall, Kerryann Foley and Junko Shigemitsu for useful conversations.

\section{References}

[1] E. Gulez et al., Phys. Rev. D 73, 074502 (2006); M. Okamoto, Proc. Sci., LAT2005 (2005) 013.

[2] J. H. Sloan, Nucl. Phys. Proc. Suppl. 63, 365 (1998); K. M. Foley and G. P. Lepage, Nucl. Phys. Proc. Suppl. 119, 635 (2002); A. Dougall et al., LAT2005 (2005) 219.

[3] K. M. Foley, Ph.D. Thesis, Cornell University (2004).

[4] A. Dougall et al., Nucl. Phys. Proc. Suppl. 140, 431 (2005).

[5] W. Dimm et al., Nucl. Phys. Proc. Suppl. 42, 403 (1995); K. J. Juge, Nucl. Phys. Proc. Suppl. 94, 584 (2001); H. D. Trottier et al., Phys. Rev. D 65, 094502 (2002); K. Y. Wong, H. D. Trottier and R. M. Woloshyn, Phys. Rev. D 73, 094512 (2006).

[6] G. M. de Divittis, R. Petronzio and N. Tantalo, Phys. Lett. B 595, 408 (2004); J. M. Flynn et al., Phys. Lett. B 632, 313 (2006).

[7] C. Bernard et al., Phys. Rev. D 64, 054506 (2001); A. Gray et al., Phys. Rev. D 64, 094507 (2005). 